Research Article

\title{
Experimental and Constitutive Model Study on Dynamic Mechanical Behavior of Metal Rubber under High-Speed Impact Loading
}

\author{
Youchun Zou $\left(\mathbb{D}\right.$, Chao Xiong $\mathbb{D}$, Junhui Yin $\mathbb{D}^{D}$, Kaibo Cui, Xiujie Zhu, Huiyong Deng, \\ and Shijun Song
}

Department of Artillery Engineering, Shijiazhuang Campus, Army Engineering University of PLA, Shijiazhuang 050003, China

Correspondence should be addressed to Chao Xiong; xiongchao@tsinghua.org.cn and Junhui Yin; yuanzhidao@163.com

Received 26 September 2020; Revised 12 January 2021; Accepted 18 February 2021; Published 28 February 2021

Academic Editor: Franck Poisson

Copyright (c) 2021 Youchun Zou et al. This is an open access article distributed under the Creative Commons Attribution License, which permits unrestricted use, distribution, and reproduction in any medium, provided the original work is properly cited.

\begin{abstract}
The development of lightweight, impact-resistant, and high energy-consuming materials is of great significance for improving the defense capabilities of military equipment. As a new type of damping material, metal rubber has demonstrated great potential for application in the field of impact protection. In this paper, the dynamic mechanical response of metal rubber under a high strain rate is studied, which provides a new idea for developing high-performance protective materials. The stress-strain curves, energy absorption performance, and wave transmission performance of metal rubber at various strain rates were investigated based on a split-Hopkinson pressure bar (SHPB) device. The dynamic stress-strain curve of metal rubber is divided into three stages: elastic stage, plastic stage, and failure stage. The optimal energy absorption efficiency is greater than 0.5 , and the maximum value can reach 0.9 . The wave transmittance is less than 0.01 . The dynamic mechanical tests have proved that metal rubber has excellent energy absorption capacity and impact resistance property. A constitutive model based on Sherwood-Frost was established to predict the dynamic mechanical behavior of metal rubber. The results of comparison between the calculation and the experiment show that the constitutive model can accurately predict the dynamic mechanical performance of metal rubber.
\end{abstract}

\section{Introduction}

In recent years, military conflicts, violent incidents, and terrorist attacks still happen occasionally. Therefore, countries all over the world are committed to conducting research of lightweight, antiexplosion, and impact-resistant materials to meet urgent needs for protection of military equipment.

At present, foamed aluminum, rubber, and fiber composites are widely used because of their good energy absorption properties. The research studies on the dynamic mechanical properties of these materials are mostly investigated based on split-Hopkinson pressure bar (SHPB) tests. Amaro et al. [1] studied the influence of structure and material on the dynamic mechanical properties of foamed aluminum. The experimental results suggested that Al6082-T4 foam and dual-size cell structure foam has excellent dynamic mechanical properties. Hu et al. [2] proposed the use of vacuum- assisted resin infusion to prepare flax fiber reinforced composites (FFRCs). The experiment used the SHPB device to impact FFRCs and evaluated the dynamic response of FFRCs from the macroscopic and microscopic failure mechanism. It was shown that FFRCs had good energy absorption performance under high-velocity impact. Li et al. [3] studied the dynamic mechanical properties and energy absorption characteristics of high-damping rubber under SHPB highspeed impact. The results revealed that the high-damping rubber had good energy absorption performance and the energy absorption ability increased with the increasing strain rate. At present, the research on the abovementioned materials has matured considerably, and it is difficult to further improve their performance. Therefore, it is necessary to develop new materials with excellent performance.

Metal rubber (MR) is a kind of porous material woven by metal wire. It is often used to reduce vibration [4]. Metal rubber (MR) combines the advantages of rubber 
and metal. The rubber polymer material has high elasticity, large damping, and strong ability to absorb energy. However, the rubber polymer materials cannot work in complex environments, such as high temperature, low temperature, strong impact, salt spray corrosion, and nuclear radiation $[5,6]$. MR not only has the advantages of rubber polymers but also demonstrates the ability of working in extreme environments. It is sometimes referred to as entangled metallic wire material (EMWM) [7-10], elastic porous wire mesh (EPWM) [11], or “metal wire mesh (MWM) [12-14].

The research on MR mostly concentrated on quasistatic mechanical properties $[15,16]$, dynamic mechanical performance [17-19], mechanical models [20-25], the influence of various factors on the mechanical performance $[5,16,26]$, and engineering applications [27, 28]. Tan et al. [8] summarized the mechanical properties of MR under quasi-static compression. MR undergoes an elastic deformation stage, strain strengthening stage, and densification stage during compression loading. Liu et al. [29] measured the impact resistance of MR by Charpy impact tests. The research indicates that the impact toughness of $\mathrm{MR}$ increases with the decrease in porosity. Wu et al. [30] studied the performance of MR under low-speed impact. The paper revealed the influence mechanism of density and strain rate on the impact behavior. Meanwhile, a constitutive model under low-velocity impact was established. Fengli et al. [23] proposed a cantilever beam model of MR. The cantilever beam model was based on the spatial shape and contact mode of the metal wire. This model can accurately predict the force-displacement curves of MR during loading and unloading. Wang et al. [31] applied MR to the cushioning device of the airborne optoelectronic pod and found the MR played an important role in suppressing vibration.

The purpose of this paper is to explore the application of metal rubber in the area of antiexplosion and impact resistance. Therefore, it is important to study the mechanical properties of MR under high-speed impact. However, there are few studies on the dynamic mechanical behavior of MR under high-speed impact loading. The high-speed impact experiments in this article will be carried out on the SHPB device. The stress-strain curves, energy absorption, and energy transmittance of $\mathrm{MR}$ at different strain rates will be studied. Finally, a constitutive model of MR under high strain rate conditions will be established.

\section{Materials and Methods}

2.1. Materials. The MR sample is shown in Figure 1. In this experiment, the MR samples were made of 304 austenitic stainless-steel wire $(0 \mathrm{Cr} 18 \mathrm{Ni} 9)$. The diameter of wire is $0.3 \mathrm{~mm}$. There are three steps to fabricate the MR specimens: (1) encircling the stainless-steel wire into a compact helix, (2) stretching and weaving the compact helix into porous base materials, and (3) putting the base materials in a designed mold and applying a compression force. In this paper, six samples with the same process parameters were prepared. The original process parameters of the samples are shown in Table 1 . The relative density in Table 1 is defined as follows:

$$
\bar{\rho}=\frac{\rho_{\mathrm{MR}}}{\rho_{\mathrm{WIRE}}}=\frac{M / V}{\rho_{\mathrm{WIRE}}}=1-\varphi,
$$

where $\rho_{\mathrm{MR}}$ is the density of MR which is calculated as the ratio between the mass $M$ and the volume $V, \rho_{\text {WIRE }}$ is the density of $0 \mathrm{Cr} 18 \mathrm{Ni} 9, \rho_{\mathrm{WIRE}}=7.89 \mathrm{~g} / \mathrm{cm}^{3}$, and $\varphi$ is the porosity of MR.

2.2. Split-Hopkinson Pressure Bar Tests. Split-Hopkinson pressure bar (SHPB) technology is an important test method for studying the dynamic mechanical performance of the materials. Figure 2 shows the SHPB dynamic testing system. The SHPB dynamic testing system is composed of a gas gun, a striker, a light beam, an incident bar, a transmitted bar, a damper, a high dynamic strain indicator, an oscilloscope, and a computer. The entire impact process is captured by a 10-bit CMOS camera (PCO.1200hs, PCO AG, Kelheim, Germany). The incident and transmitted bars are made of aluminum 7075 with a diameter of $40 \mathrm{~mm}$, its elastic modulus is $71 \mathrm{GPa}$, and its density is $2.81 \mathrm{~g} / \mathrm{cm}^{3}$. The lengths of the incident bar and transmitted bar are both $2 \mathrm{~m}$. As shown in Figure 2(b), the specimen was sandwiched between the SHPB device.

The gas gun launches the striker, and the striker impacts on the incident bar to generate an elastic incident wave $\varepsilon_{I}$. The elastic incident wave propagates through the incident bar and reaches the interface between the incident bar and sample. A part of the incident wave is reflected as reflected wave $\varepsilon_{R}$, and another part wave is transmitted through the specimen as transmitted wave $\varepsilon_{T}$.

The incident and reflected waves are recorded by the strain gauges attached on the incident bar. The transmission wave is weak due to the small wave impedance of MR.

Due to the high sensitivity of the semiconductor strain gauge, it is used to record signals on the transmission bar [32]. The signals collected by strain gauges are analyzed by a high dynamic strain indicator and recorded by an oscilloscope.

According to the stress wave theory, $\sigma(t), \varepsilon(t)$, and $\dot{\varepsilon}(t)$ should satisfy the following equations:

$$
\begin{aligned}
& \sigma(t)=\frac{A E}{2 A_{s}}\left[\varepsilon_{I}(t)+\varepsilon_{R}(t)+\varepsilon_{T}(t)\right], \\
& \varepsilon(t)=\frac{C}{L_{s}} \int_{0}^{t}\left[\varepsilon_{I}(t)-\varepsilon_{R}(t)-\varepsilon_{T}(t)\right] \mathrm{d} t, \\
& \dot{\varepsilon}(t)=\frac{C}{L_{s}}\left[\varepsilon_{I}(t)-\varepsilon_{R}(t)-\varepsilon_{T}(t)\right],
\end{aligned}
$$

where $A, E$, and $C$ are the cross-section area, the elastic modulus, and stress wave velocity of the bar, respectively. $\varepsilon_{I}(t), \varepsilon_{R}(t)$, and $\varepsilon_{T}(t)$ are the incident strain signal, reflected strain signal, and transmitted strain signal, respectively. $A_{s}$ and $L_{s}$ are the cross-sectional area and thickness of the 


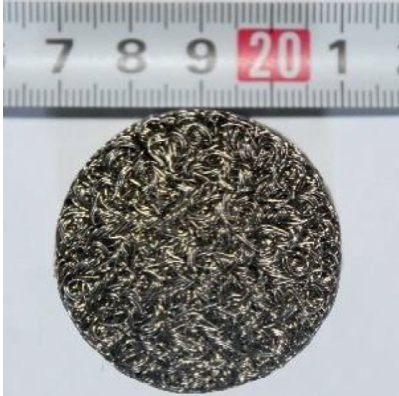

(a)

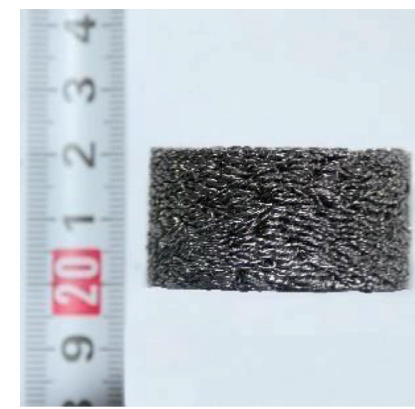

(b)

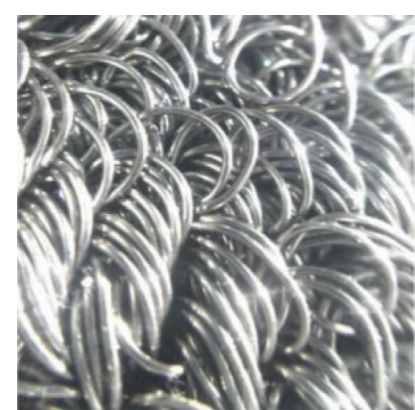

(c)

Figure 1: (a) Non molding direction of MR; (b) Molding direction of MR; (c) Magnified view of MR.

TABLE 1: Original process parameters of MR specimens

\begin{tabular}{lcccc}
\hline Specimen number & Mass $(\mathrm{g})$ & Original height $(\mathrm{mm})$ & Original diameter $(\mathrm{mm})$ & Relative density \\
\hline MR-1 & 60.2 & 20.45 & 39.74 & 39.76 \\
MR-2 & 60.4 & 20.42 & 39.72 & 0.300 \\
MR-3 & 60.2 & 20.43 & 39.75 & 0.301 \\
MR-4 & 60.1 & 20.46 & 39.74 & 0.300 \\
MR-5 & 60.2 & 20.45 & 39.77 & 0.300 \\
MR-6 & 60.2 & 20.44 & & 0.300 \\
\hline
\end{tabular}

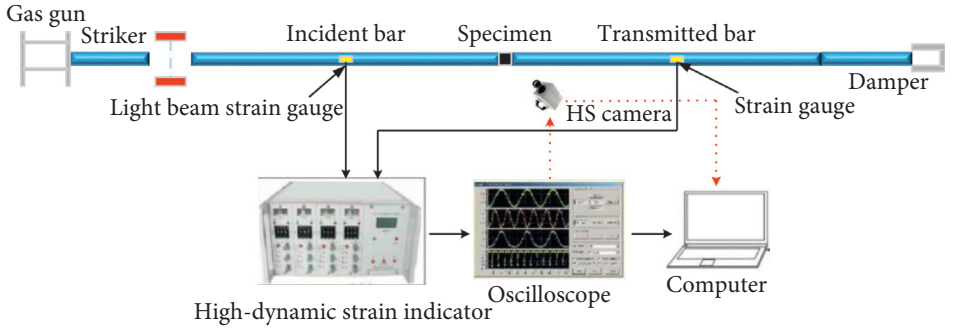

(a)

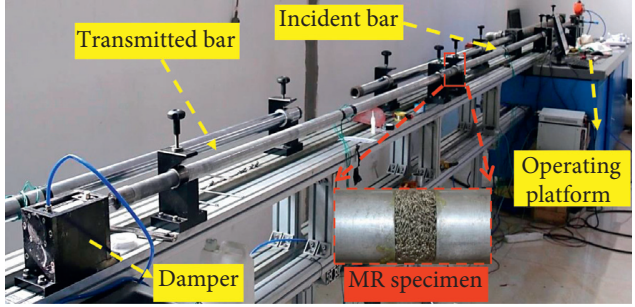

(b)

FIgURE 2: Diagram of the SHPB device.

sample, respectively. According to the stress equilibrium of the sample, the strain satisfies the following equation:

$$
\varepsilon_{I}(t)+\varepsilon_{R}(t)=\varepsilon_{T}(t) .
$$

Substituting (5) into (2)-(4), $\sigma(t), \varepsilon(t)$, and $\dot{\varepsilon}(t)$ can be simplified as follows:

$$
\begin{aligned}
& \sigma(t)=\frac{A E}{A_{s}} \varepsilon_{T}(t), \\
& \varepsilon(t)=\frac{-2 C}{L_{s}} \int_{0}^{t} \varepsilon_{R}(t) \mathrm{d} t, \\
& \dot{\varepsilon}(t)=\frac{-2 C}{L_{s}} \varepsilon_{R}(t) .
\end{aligned}
$$

The strain rates of this test are shown in Table 2. Figure 3 shows the representative images of incident, reflected, and transmitted signals in the experiment.

\section{Results and Discussion}

3.1. Stress-Strain Curve Characteristics. Figure 4 is a typical stress-strain curve and the impact process of MR. The typical stress-strain curve of MR can be divided into three stages: (1) elastic stage, (2) plastic stage, and (3) failure stage.

At the stage I, the metal wires contact each other and deform. The deformation of the metal wire is small and within the elastic range. The MR can be equivalent to the superposition of all metal wires, so the MR is also within the elastic range. Therefore, the stress-strain curve is approximately linear.

As the stress increases, the MR enters the plastic stage. In the plastic stage, the metal wires undergo plastic deformation, and relative sliding occurs between the metal wires. The internal structure of MR is compact. Although the metal wire slides, it is still constrained by other metal wires and space, and the MR still has load-bearing capacity.

In the plastic stage, the stress slowly rises to the maximum. 
TABLE 2: Strain rate of the samples.

\begin{tabular}{lcccccc}
\hline Specimen number & MR-1 $(\mathrm{s})$ & MR-2 $(\mathrm{s})$ & MR-3 (s) & MR-4 (s) & MR-5 (s) & MR-6 (s) \\
\hline Strain rate & $630 \mathrm{~s}^{-1}$ & $1160 \mathrm{~s}^{-1}$ & $1410 \mathrm{~s}^{-1}$ & $1630 \mathrm{~s}^{-1}$ & $1770 \mathrm{~s}^{-1}$ & $1840 \mathrm{~s}^{-1}$ \\
\hline
\end{tabular}

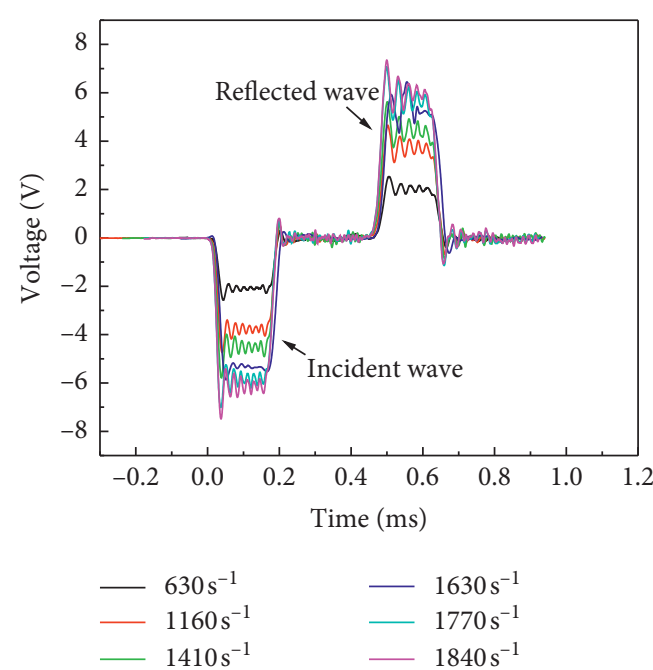

(a)

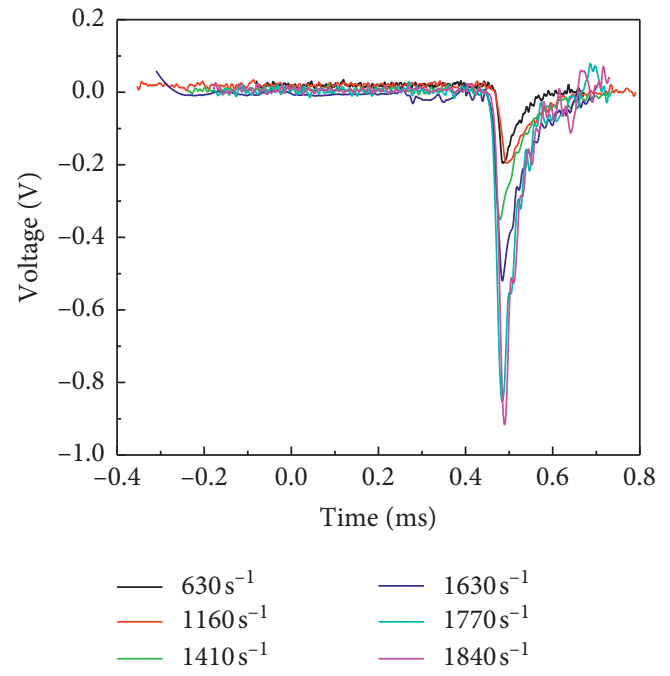

(b)

FIGURE 3: (a) Incident and reflected signals; (b) transmitted signals.
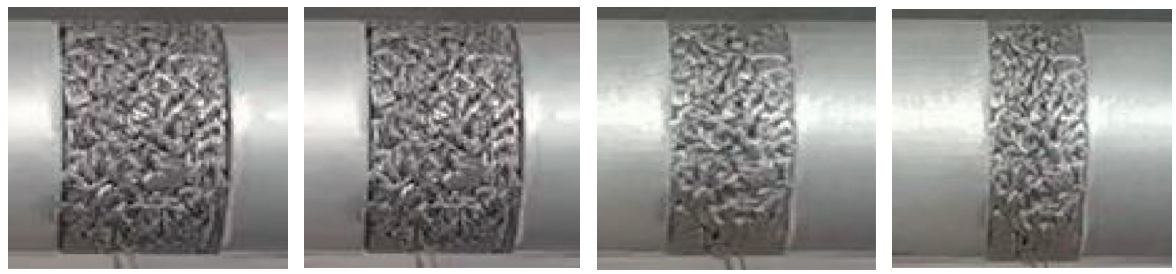

(a)

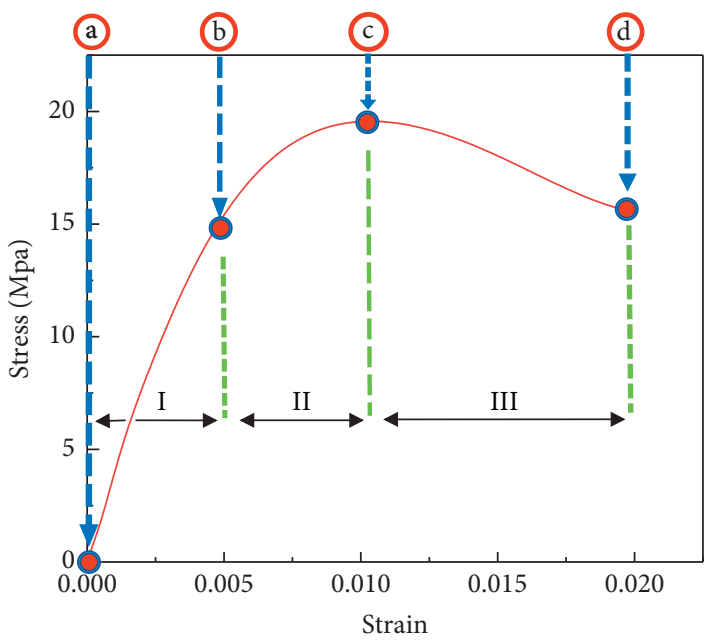

(b)

Figure 4: Typical stress-strain curve and the impact process of MR.

When the stress increases beyond the ultimate load-bearing capacity, the MR enters the failure stage. During the failure stage, the metal wire will lose stability, resulting in unrecoverable plastic deformation, and the load-bearing capacity will decrease.
Figure 5 shows the stress-strain curves of MR at different strain rates. It can be seen from Figure 5 that the elastic modulus increases as the strain rate increases. In the elastic stage, it can be known from Hooke's law $F=k x$ that the 


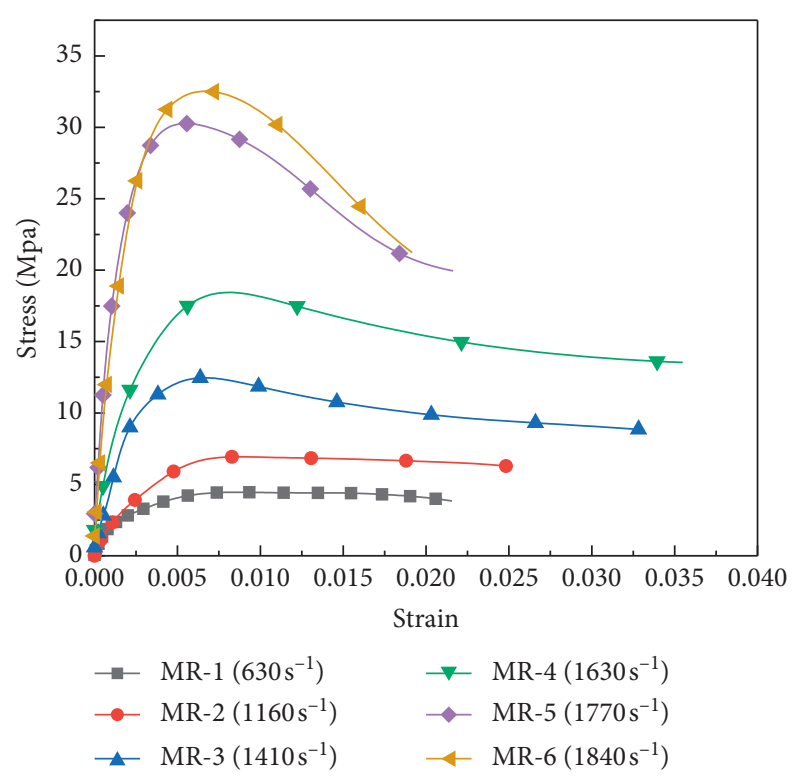

Figure 5: Stress-strain curves at different strain rates.

greater the force is, the greater the elastic modulus is. The stress in the failure stage decreases faster as the strain rate increases. It is because the greater the strain rate, the faster the MR failure, and the faster the stress drop.

When the external load exceeds the critical load of MR, the plastic deformation occurs. Table 3 shows the diameter and thickness of MR after impact. Because the strain rates of MR-1 and MR-2 are small, the thickness and diameter change little compared to before. The MR-3, MR-4, MR-5, and MR-6 show obvious plastic deformation. The height of specimens decreases as the strain rate increases. The MR- 5 and MR- 6 not only decrease in height but also increase in diameter.

The peak stress is the maximum stress of the material which represents the ultimate load-bearing capacity of the material. The peak stress of MR and the result of fitting the function are shown in Figure 6. The relationship between the strain rate and peak stress is defined by an exponential function. Therefore, the ultimate load-bearing capacity of MR increases with the increase in the strain rate. The reason for this phenomenon is that a higher strain rate makes MR denser. The increase in the density of MR leads to increase in its ultimate load-bearing capacity.

3.2. Energy Absorption Characteristics. This paper uses three evaluation methods to assess the energy absorption performance of MR.

3.2.1. Energy Absorption. The energy absorption can evaluate the energy absorption capacity of the material. The area value under the stress-strain curve which is calculated by the integral of the curve represents the energy absorbed by the unit volume of the material. Energy absorbed by the unit volume of the material is represented by $Q$ which is defined as follows [33]:
TABle 3: Specimen size parameters after impact.

\begin{tabular}{lcc}
\hline Specimen number & $\begin{array}{c}\text { Height after } \\
\text { impact }(\mathrm{mm})\end{array}$ & Diameter after impact $(\mathrm{mm})$ \\
\hline MR-1 & 20.00 & 40.00 \\
MR-2 & 19.99 & 40.00 \\
MR-3 & 19.71 & 40.01 \\
MR-4 & 19.46 & 40.03 \\
MR-5 & 18.82 & 40.69 \\
MR-6 & 18.79 & 40.71 \\
\hline \multicolumn{2}{c}{} \\
& &
\end{tabular}

where $\varepsilon_{i}$ is any strain.

3.2.2. Energy Absorption Efficiency. The expression of the energy absorption efficiency $E$ is [34] as follows:

$$
E=\frac{\int_{0}^{\varepsilon_{i}} \sigma \mathrm{d} \varepsilon}{\sigma_{i}}=\frac{Q}{\sigma_{i}},
$$

where $\varepsilon_{i}$ is any strain and $\sigma_{i}$ is the stress corresponding to the strain. The larger the value of $E$ means that the material absorbs more energy at low stress level, avoiding damage to the material under high stress.

3.2.3. Optimal Energy Absorption Efficiency. The optimal energy absorption efficiency is the ratio of the actual energy absorbed by the material to the energy absorbed by the material under ideal conditions. The optimal energy absorption efficiency $I$ is defined as follows [35]:

$$
I=\frac{\int_{0}^{\varepsilon_{i}} \sigma \mathrm{d} \varepsilon}{\sigma_{i} \times \varepsilon_{i}}=\frac{E}{\varepsilon_{i}}=\frac{Q}{\sigma_{i} \times \varepsilon_{i}},
$$




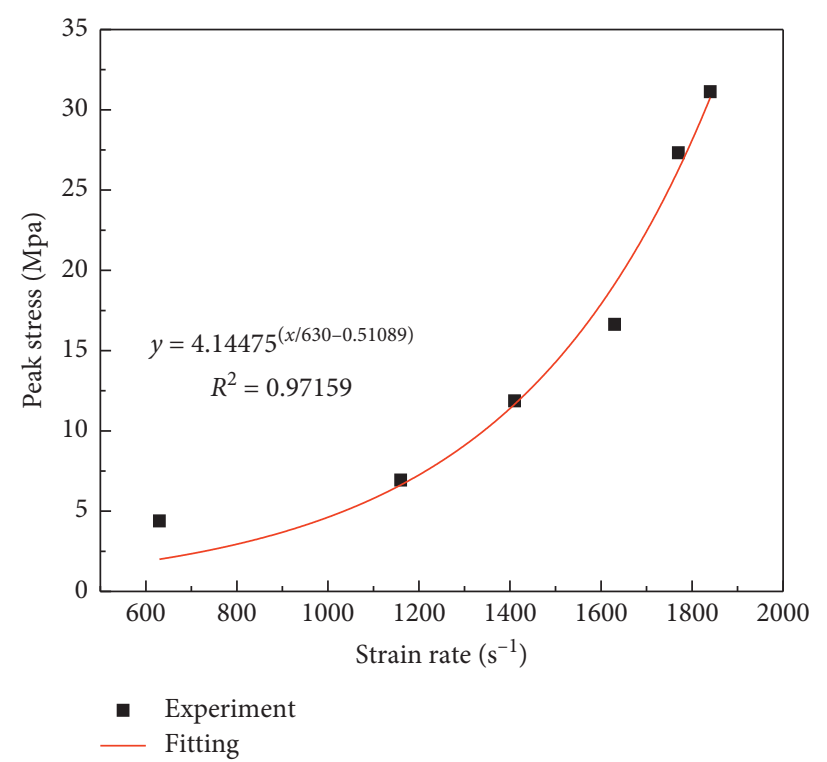

FIGURE 6: Peak stress at different strain rates.

where $\varepsilon_{i}$ is any strain and $\sigma_{i}$ is the stress corresponding to the strain.

From Figure 4, it is known the stress-strain curve of MR has a failure stage. The stress in the failure stage decreases as the strain increases. In this case, the optimal energy absorption efficiency of MR calculated by formula (9) will be greater than 1 . This conclusion is undesirable. Therefore, we have revised the definition of the optimal energy absorption efficiency and modified $\sigma_{i}$ to $\sigma_{\max }$. The optimal energy absorption efficiency $I$ is refined as

$$
I=\frac{\int_{0}^{\varepsilon_{i}} \sigma \mathrm{d} \varepsilon}{\sigma_{\max } \times \varepsilon_{i}}=\frac{Q}{\sigma_{\max } \times \varepsilon_{i}},
$$

where $\varepsilon_{i}$ is any strain and $\sigma_{\max }$ is the maximum stress within the limits of the integral. The modified optimal energy absorption efficiency is more reasonable to assess the energy absorption performance of MR.

Figure 7 is the $Q-\varepsilon$ curves of $M R$ at different strain rates. The energy absorption of MR increases with increasing strain. Under the same strain, the MR with a high strain rate absorbs more energy.

Figures $8(\mathrm{a})$ and $8(\mathrm{~b})$ are the $E-\varepsilon$ and $E-\sigma$ curves of MR under different strain rates. From Figure 8(a), it can be concluded that the energy absorption efficiency of the samples has little difference in the initial strain range. As the strain increases, the gap in energy absorption efficiency becomes larger. In general, the energy absorption efficiency of MR increases as the strain rate increases. Figure $8(\mathrm{~b})$ is the $E-\sigma$ curve. Under the same stress, the MR with low strain rate has greater energy absorption efficiency. This is because the samples with the lower strain rates absorb more energy under the same stress condition.

Figure 9 shows the $I-\varepsilon$ curves. The optimal energy absorption efficiency of MR under different strain rates is always greater than 0.5 , and the maximum value is close to
0.9 , which indicates that $\mathrm{MR}$ is a material with good energy absorption properties.

3.3. Wave Transmission Performance. The high-speed impact test of MR involves a variety of energy conversion. Studying the energy transfer process is of great significance for evaluating the energy absorption characteristics of MR. Based on the conservation of energy, the incident energy, reflected energy, transmitted energy, and dissipated energy are expressed as follows:

$$
\begin{aligned}
& W_{I}=\frac{A C}{E} \int_{0}^{T} \sigma_{I}^{2}(t) \mathrm{d} t=A C E \int_{0}^{T} \varepsilon_{I}^{2}(t) \mathrm{d} t, \\
& W_{R}=\frac{A C}{E} \int_{0}^{T} \sigma_{R}^{2}(t) \mathrm{d} t=A C E \int_{0}^{T} \varepsilon_{R}^{2}(t) \mathrm{d} t, \\
& W_{T}=\frac{A C}{E} \int_{0}^{T} \sigma_{T}^{2}(t) \mathrm{d} t=A C E \int_{0}^{T} \varepsilon_{T}^{2}(t) \mathrm{d} t, \\
& W=W_{I}-W_{R}-W_{T},
\end{aligned}
$$

where $W_{I}, W_{R}, W_{T}$, and $W$ are incident, reflected, transmitted, and dissipated energy, respectively. $A, C$, and $E$ are the cross-sectional area, stress wave velocity, and elastic modulus of the bar, respectively. $\sigma_{I}, \sigma_{R}, \sigma_{T}, \varepsilon_{I}, \varepsilon_{R}$, and $\varepsilon_{T}$ are the stress and strain corresponding to the incident, reflected, and transmitted wave, and $T$ is the loading time.

Figure $10(a)$ is the energy-strain rate curves. As the incident energy increases, the reflected energy, the dissipated energy, and the transmitted energy all increase. Figure 10(b) is the ratio of incident, reflected, and dissipated energy to the incident energy. From Figure 10(b), it can be concluded that most of the incident energy is reflected, part of the incident energy is dissipated, and only a very small part of the incident energy is transmitted. The transmittance always remained below 0.01. It is because that $M R$ is a porous 


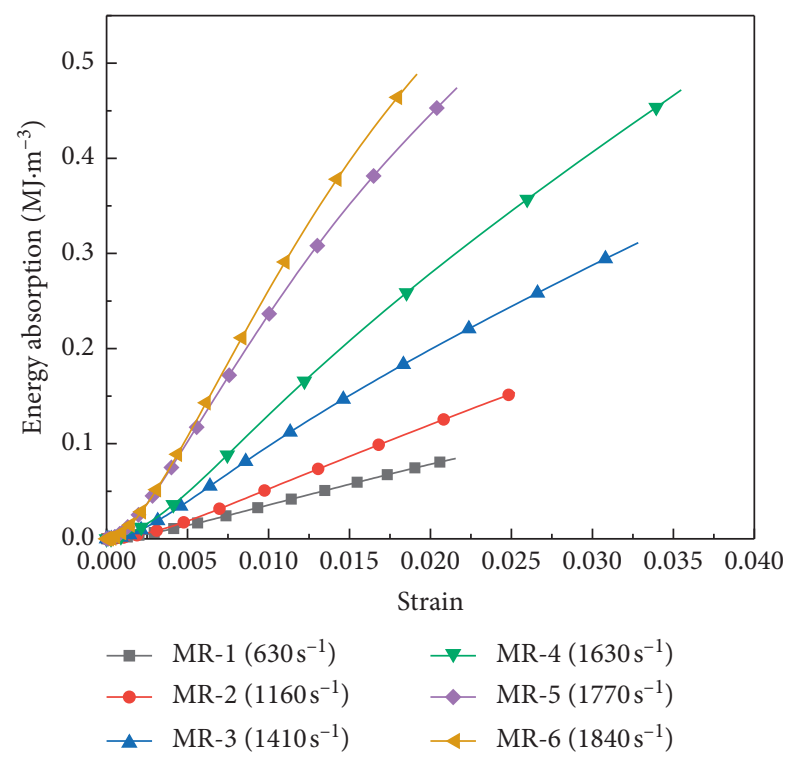

FigURE 7: $Q-\varepsilon$ curves.

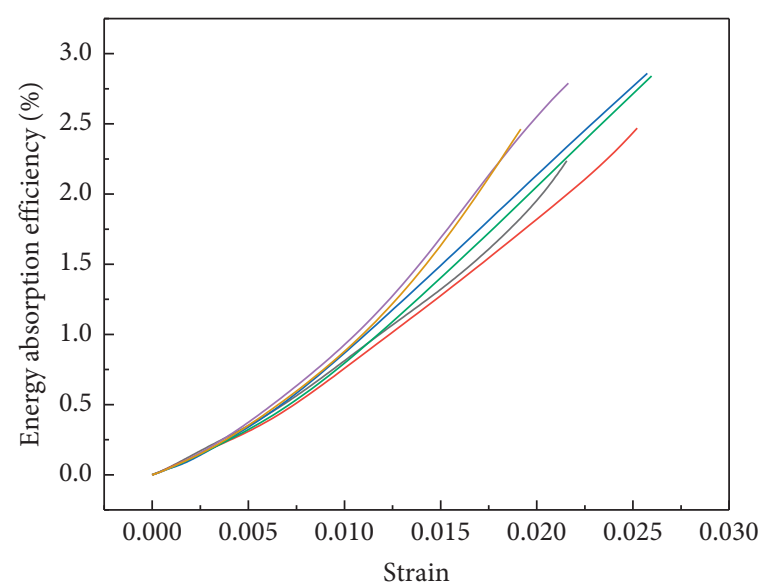

$\begin{array}{ll}-\operatorname{MR}-1\left(630 \mathrm{~s}^{-1}\right) & -\operatorname{MR}-4\left(1630 \mathrm{~s}^{-1}\right) \\ \text { MR-2 }\left(1160 \mathrm{~s}^{-1}\right) & -\operatorname{MR}-5\left(1770 \mathrm{~s}^{-1}\right) \\ \text { MR-3 }\left(1410 \mathrm{~s}^{-1}\right) & -\operatorname{MR}-6\left(1840 \mathrm{~s}^{-1}\right)\end{array}$

(a)

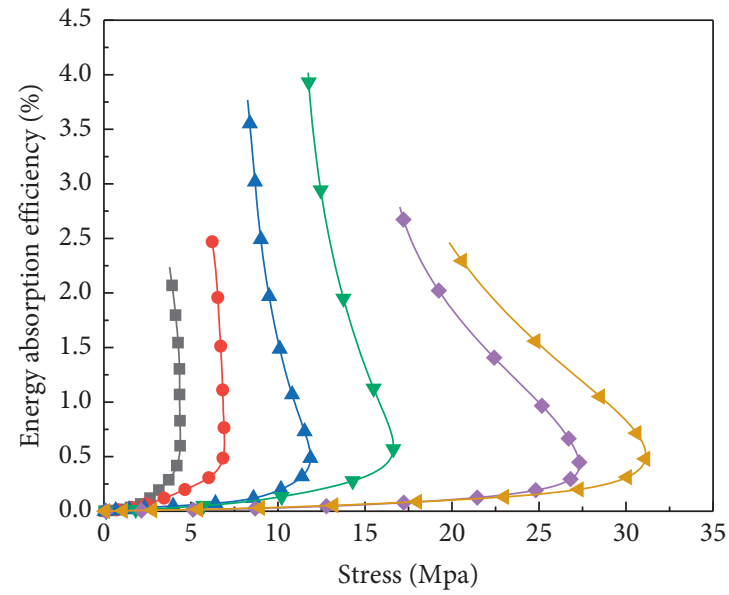

$$
\begin{array}{ll}
\longrightarrow \operatorname{MR}-1\left(630 \mathrm{~s}^{-1}\right) & \rightarrow \operatorname{MR}-4\left(1630 \mathrm{~s}^{-1}\right) \\
\multimap \operatorname{MR}-2\left(1160 \mathrm{~s}^{-1}\right) & \multimap \operatorname{MR}-5\left(1770 \mathrm{~s}^{-1}\right) \\
\leftarrow \operatorname{MR}-3\left(1410 \mathrm{~s}^{-1}\right) & \longrightarrow \operatorname{MR}-6\left(1840 \mathrm{~s}^{-1}\right)
\end{array}
$$

(b)

Figure 8: (a) $E-\varepsilon$ curves; (b) $E-\sigma$ curves.

material, and the stress wave is reflected multiple times in the irregular pores. During the multiple reflection process, most of the stress wave is reflected, part of the stress wave is consumed, and only a very small part of the stress wave can pass through the MR. Therefore, MR has excellent stress wave dissipation performance.

\section{Constitutive Model Established}

From the experimental results above, it is shown that the dynamic mechanical behavior of MR at high strain rates is complicated. In this paper, a constitutive model of MR is established based on the Sherwood-Frost equation. The commonly used Sherwood-Frost constitutive model for porous metal materials is as follows:

$$
\sigma(\varepsilon)=H(T) G(\rho) M(\varepsilon, \dot{\varepsilon}) f(\varepsilon),
$$

where $\sigma(\varepsilon)$ is the stress, $H(T)$ is the temperature function, $G(\rho)$ is the density function, $M(\varepsilon, \dot{\varepsilon})$ is the strain rate function, and $f(\varepsilon)$ is the shape function.

4.1. Fitting of Shape Functions. The $\sigma-\varepsilon$ curves at different strain rates show different features. Therefore, it is unreasonable to use only one shape function to calculate the constitutive model. It is necessary to classify the $\sigma-\varepsilon$ curves 


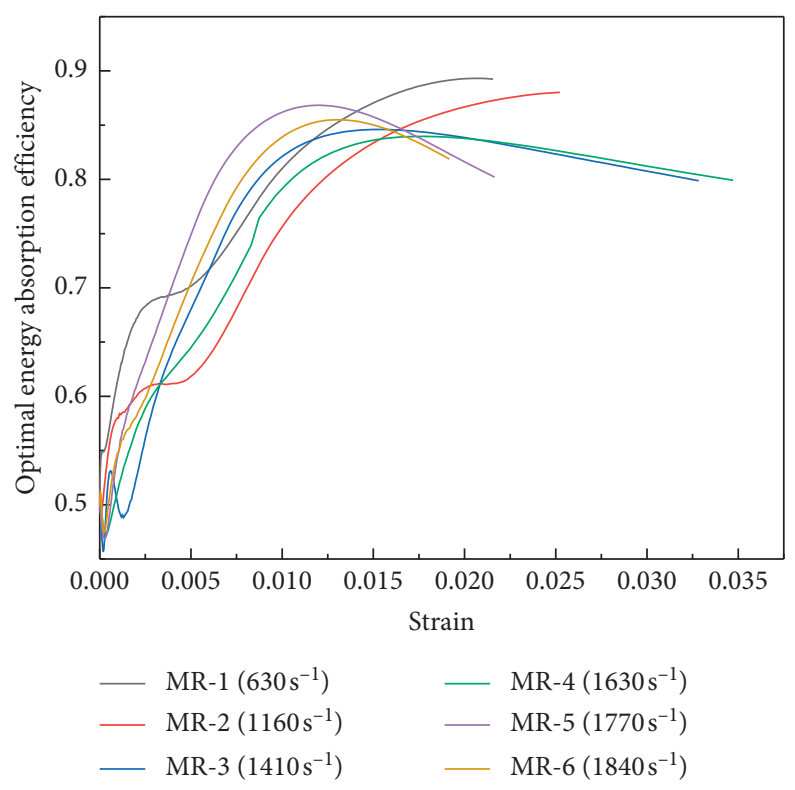

FIGURE 9: $I-\varepsilon$ curves.

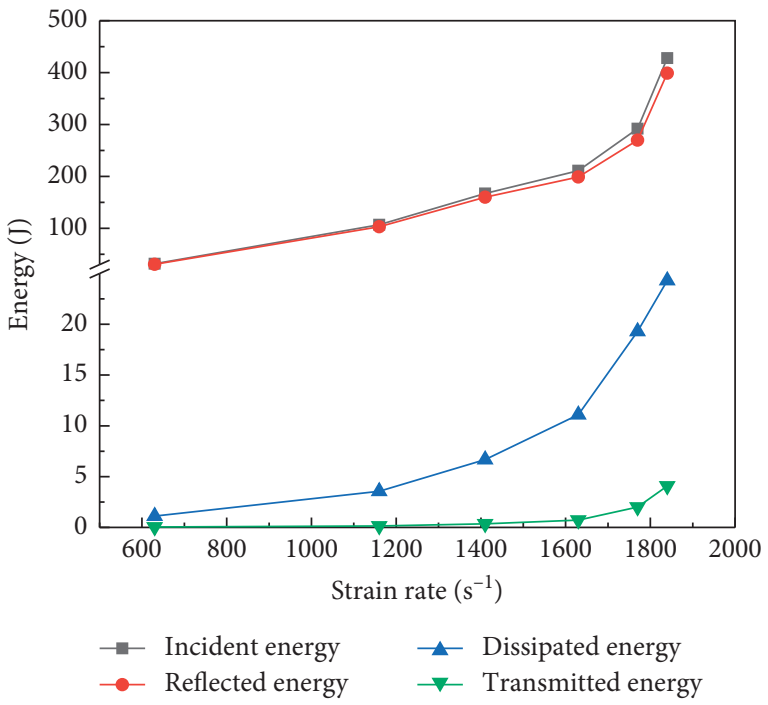

(a)

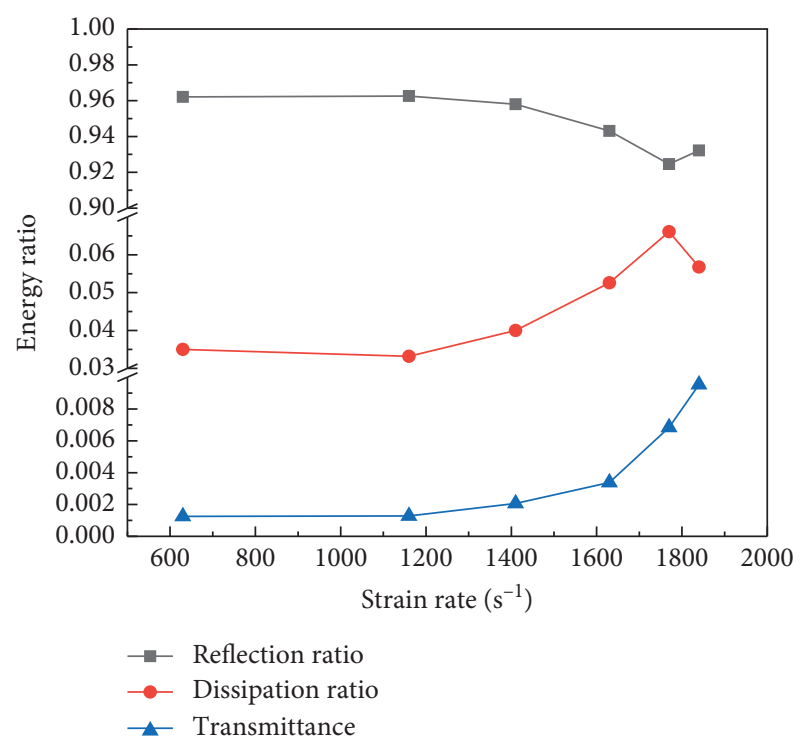

(b)

Figure 10: (a) Incident energy, reflected energy, dissipated energy, and transmitted energy at different strain rates; (b) energy ratio at different strain rates.

according to its characteristics and use a shape function in the stress-strain curves of the same feature. In this paper, it divided the strain rate range into three regions according to the features of the stress-strain curves in Figure 4. The strain rate from $630 \mathrm{~s}^{-1}$ to $1410 \mathrm{~s}^{-1}$ is the first region, $1410 \mathrm{~s}^{-1}$ to $1770 \mathrm{~s}^{-1}$ is the second region, and $1770 \mathrm{~s}^{-1}$ to $1840 \mathrm{~s}^{-1}$ is the third region. Within each strain rate region, it is considered that the characteristics of the stress-strain curves are to be similar. In each region, a reference curve is selected to calculate the shape function. In this paper, the $\sigma-\varepsilon$ curves at strain rates of $630 \mathrm{~s}^{-1}, 1410 \mathrm{~s}^{-1}$, and $1770 \mathrm{~s}^{-1}$ are selected as reference curves to calculate the shape functions of the three regions. The shape function expression is

$$
f(\varepsilon)=\sum_{i=1}^{n} \frac{A_{i} \varepsilon^{i}}{\sigma_{0}}
$$

where $\sum_{i=1}^{n} A_{i} \varepsilon^{i}$ is the polynomial fitting term of the reference curves in each region, $n=10 ; A_{i}$ is the fitting parameter; and $\sigma_{0}$ is the peak stress of the reference curves. The $\sigma_{0}$ of the three regions are $4.39 \mathrm{MPa}, 11.87 \mathrm{MPa}$, and $27.32 \mathrm{MPa}$, respectively. The fitting results are shown in Table 4. 
TABLE 4: Fitting results.

Fitting results of stress-strain curve with a strain rate of $630 \mathrm{~s}^{-1}$

$A_{1}$

$3.68 \times 10^{3}$

$A_{6}$

$-2.61 \times 10^{15}$

$R^{2}=0.9998$

Fitting results of stress-strain curve with a strain rate of $1410 \mathrm{~s}^{-1}$
$A_{3}$

$8.98 \times 10^{8}$

$A_{8}$
$-5.36 \times 10^{18}$

$A_{4}$

$-2.06 \times 10^{11}$

$A_{9}$

$1.08 \times 10^{20}$

$A_{3}$

$-2.47 \times 10^{8}$

$A_{8}$

$5.04 \times 10^{17}$
$A_{2}$
$04 \times 10^{5}$

$A_{7}$
$-99 \times 10^{16}$

$4.74 \times 10^{14}$

$R^{2}=0.99854$

Fitting results of stress-strain curve with a strain rate of $1770 \mathrm{~s}^{-1}$
$A_{3}$

$1.13 \times 10^{9}$

$A_{8}$

$-9.59 \times 10^{17}$
$\mathrm{A}_{2}$

$A_{7}$

$-8.55 \times 10^{14}$

$R^{2}=0.99955$

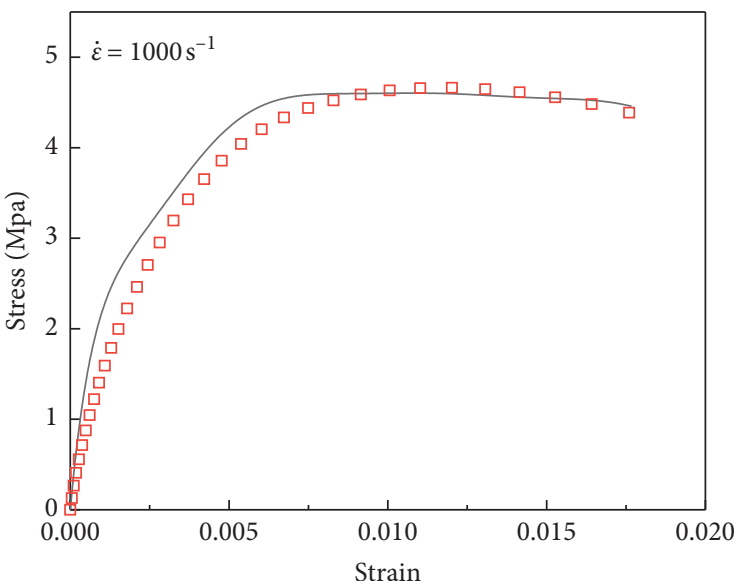

- Prediction

- Experiment

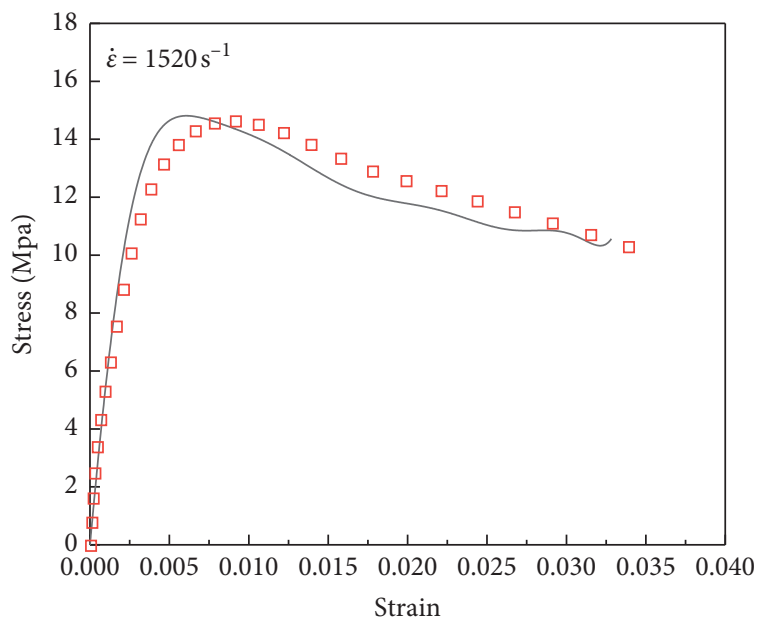

Prediction

ㅁ Experiment

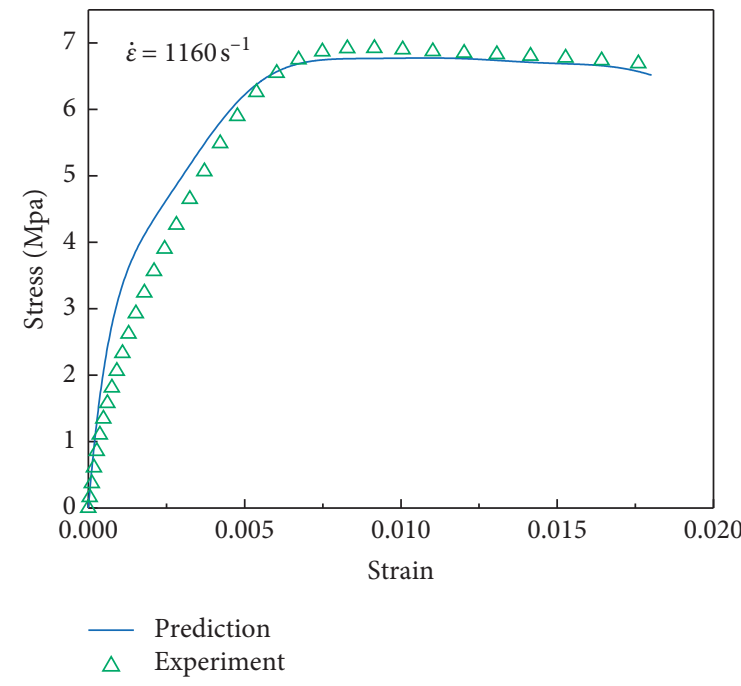

(a)

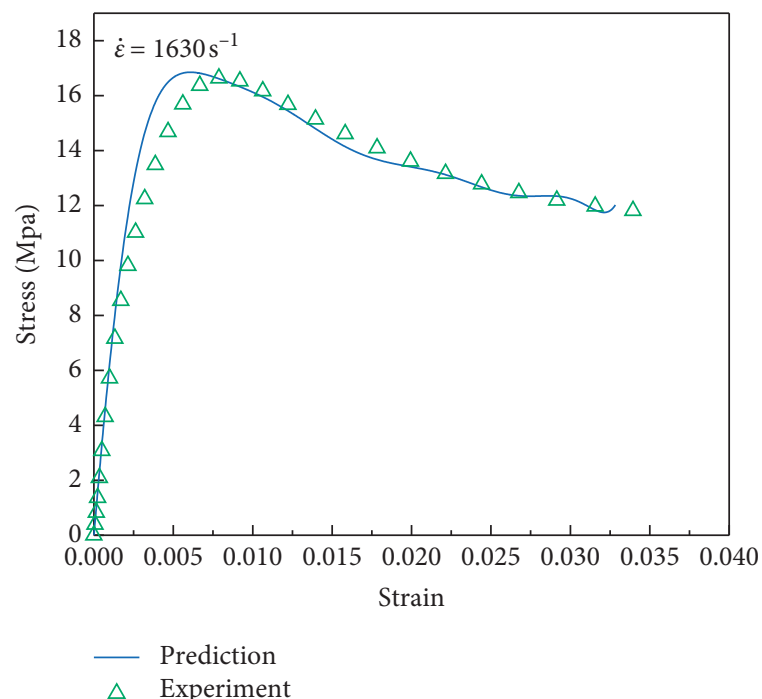

(b)
$A_{5}$ $1.35 \times 10^{13}$

$A_{10}$ $-7.84 \times 10^{19}$
$A_{5}$ $2.90 \times 10^{13}$ $A_{10}$ $-9.36 \times 10^{20}$

$A_{5}$ $-6.93 \times 10^{12}$ $A_{10}$ $4.27 \times 10^{19}$

$.47 \times 10^{11}$

$A_{9}$

$1.38 \times 10^{19}$ 

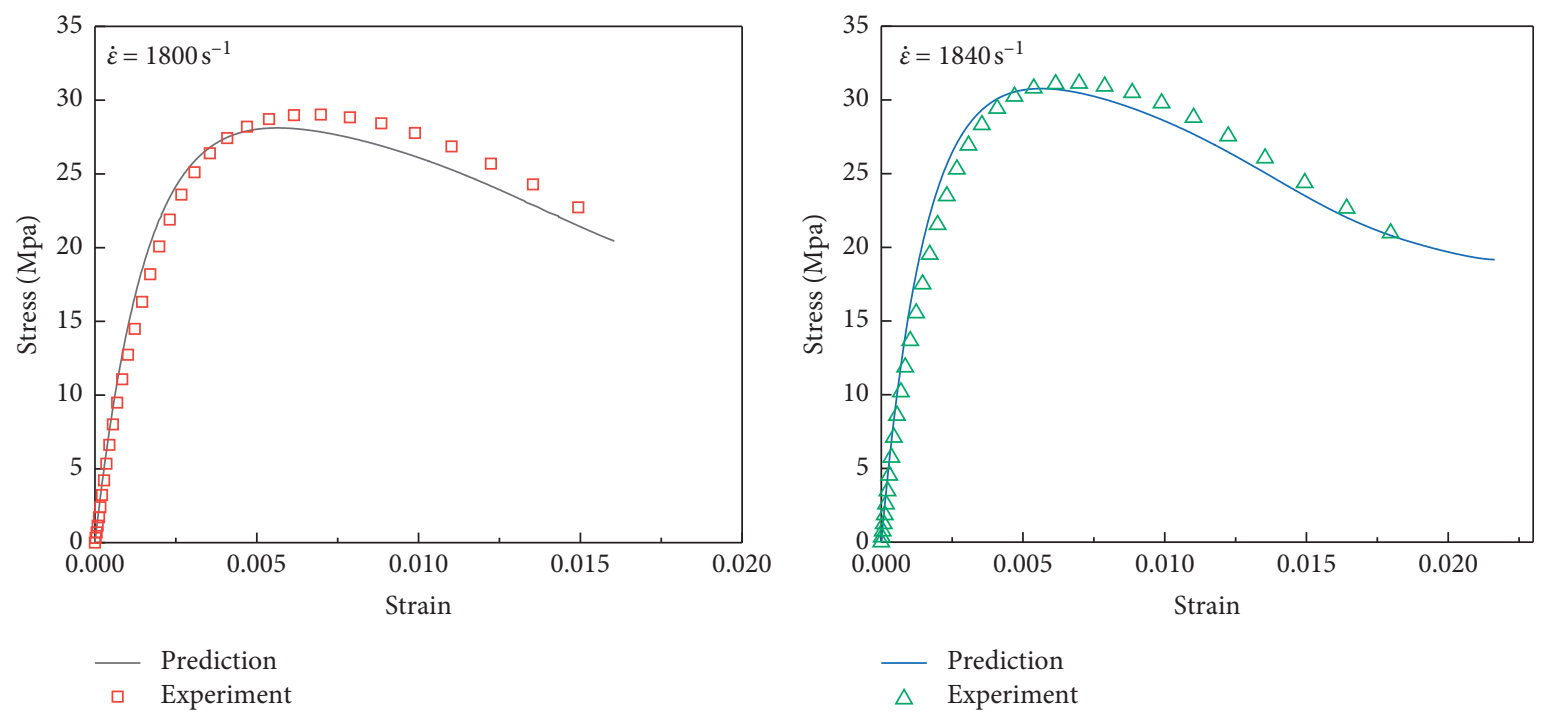

(c)

Figure 11: The comparison between the calculated results and the experimental results: (a) $\dot{\varepsilon}=1000 \mathrm{~s}^{-1}$ and $\dot{\varepsilon}=1160 \mathrm{~s}^{-1}$ in the first region; (b) $\dot{\varepsilon}=1520 \mathrm{~s}^{-1}$ and $\dot{\varepsilon}=1630 \mathrm{~s}^{-1}$ in the second region; (c) $\dot{\varepsilon}=1800 \mathrm{~s}^{-1}$ and $\dot{\varepsilon}=1840 \mathrm{~s}^{-1}$ in the third region.

4.2. Influence of Strain Rate. As shown in Figure 6, the relationship between strain rate and peak stress is approximately an exponential function. Therefore, the relationship between the strain rate and the peak stress in each region is also an exponential function. After analysis and comparison, $M(\varepsilon, \dot{\varepsilon})$ is defined as

$$
M(\varepsilon, \dot{\varepsilon})=A^{\left(\left(\dot{\varepsilon} / \varepsilon_{0}\right)+B\right)},
$$

where $A$ and $B$ are the fitting parameters and $\varepsilon_{0}=630 \mathrm{~s}^{-1}$. The strain rate item obtained through fitting can be expressed as

$$
M(\varepsilon, \dot{\varepsilon})=4.14475^{((\dot{\varepsilon} / 630)-0.51089)} .
$$

4.3. Establish and Verify the Constitutive Model. The constitutive model of MR calculated from formulas (12)-(15) can be expressed as

$$
\begin{aligned}
& \sigma(\varepsilon)=\left(4.14475^{((\dot{\varepsilon} / 630)-0.51089)}\right)\left(\sum_{i=1}^{n} \frac{A_{i} \varepsilon^{i}}{4.39}\right), \quad 630 s^{-1}<\varepsilon<1410 s^{-1}, \\
& \sigma(\varepsilon)=\left(4.14475^{((\dot{\varepsilon} / 630)-0.51089)}\right)\left(\sum_{i=1}^{n} \frac{A_{i} \varepsilon^{i}}{11.87}\right), \quad 1410 s^{-1}<\varepsilon<1770 s^{-1}, \\
& \sigma(\varepsilon)=\left(4.14475^{((\dot{\varepsilon} / 630)-0.51089)}\right)\left(\sum_{i=1}^{n} \frac{A_{i} \varepsilon^{i}}{27.32}\right), \quad 1770 s^{-1}<\varepsilon<1840 s^{-1} .
\end{aligned}
$$

$A_{i}$ is shown in Table 4 . This article does not consider the influence of temperature and density on $\mathrm{MR}, H(T)=1 G$ $(\rho)=1$.

To examine the accuracy of the constitutive model, the dynamic experiments with strain rates of $1000 \mathrm{~s}^{-1}, 1520 \mathrm{~s}^{-1}$, and $1800 \mathrm{~s}^{-1}$ were supplemented. Figure 11 shows the comparison between the calculated results and the experimental results. By comparing experimental data and calculated data, it indicates that the fitted curves match well with the experimental results. The constitutive model can accurately predict the dynamic mechanical properties of MR under high-speed impact.

\section{Conclusions}

To study the dynamic mechanical behavior of MR, a group of SHPB high-velocity impact experiments were conducted to study the dynamic mechanical behavior of MR. The stressstrain characteristics, energy absorption characteristics, and wave transmission characteristics of MR were studied. The 
constitutive model of MR was established to predict the dynamic compression performance. The main conclusions are as follows:

(1) The dynamic stress-strain curve of MR has three stages: elastic stage, plastic stage, and failure stage. As the strain rate increases, the ultimate load-bearing capacity of MR increases.

(2) The energy absorption, energy absorption efficiency, and ideal energy absorption efficiency of MR show an obvious strain rate effect. The ideal energy absorption efficiency of MR is always greater than 0.5 , and the maximum value can reach 0.9 , which indicates that $\mathrm{MR}$ has good energy absorption performance.

(3) As the strain rate increases, the energy transmittance of the MR always remains below 0.01 , indicating that MR has excellent impact resistance performance.

(4) The established constitutive model can exactly predict the dynamic compression properties of the MR.

\section{Data Availability}

The data used to support the findings of this study are available from the corresponding author upon request.

\section{Disclosure}

This research received no external funding.

\section{Conflicts of Interest}

The authors declare that there are no conflicts of interest.

\section{Authors' Contributions}

Y.Z. and J.Y. conceptualized the study. Y.Z. and K.C. analyzed using software. Y.Z., X.Z., and H.D. validated the study. Y.Z. and S.S. did formal analysis. C.X. obtained the resources and administrated the project. Y.Z. prepared the methodology, did data curation, investigated the study, wrote the original draft, reviewed and edited the manuscript, and visualized the study. J.Y. supervised the study.

\section{References}

[1] A. M. Amaro, M. A. Neto, J. S. Cirne, and P. N. B. Reis, "Mechanical characterization of different aluminium foams at high strain rates," Materials, vol. 12, no. 9, p. 1428, 2019.

[2] D. Hu, L. Dang, C. Zhang, and Z. Zhang, "Mechanical behaviors of flax fiber-reinforced composites at different strain rates and rate-dependent constitutive model," Materials, vol. 12 , no. 6 , p. 854, 2019.

[3] X. Li, H. Mao, K. Xu, and C. Miao, "A SHPB experimental study on dynamic mechanical property of high-damping rubber," Shock and Vibration, vol. 2018, Article ID 3128268, 10 pages, 2018.

[4] Y. Ma, F. Scarpa, D. Zhang, B. Zhu, L. Chen, and J. Hong, "A nonlinear auxetic structural vibration damper with metal rubber particles," Smart Materials and Structures, vol. 22, no. 8, Article ID 084012, 2013.
[5] H. Zuo, Y. H. Chen, H. B. Bai, and H. Sun, "The compression deformation mechanism of a metallic rubber," International Journal of Mechanics and Materials in Design, vol. 2, no. 3-4, pp. 269-277, 2005.

[6] H. Junfang, "Experimental investigation on fatigue characteristics of metal-rubber material at high \& low temperature," Aerospace Materials \& Technology, vol. 37, no. 2, pp. 77-80, 2007.

[7] D. Rodney, B. Gadot, O. R. Martinez, S. R. Du Roscoat, and L. Orgéas, "Reversible dilatancy in entangled single-wire materials," Nature Materials, vol. 15, no. 1, pp. 72-77, 2016.

[8] P. Liu, Q. Tan, L. Wu, and G. He, "Compressive and pseudoelastic hysteresis behavior of entangled titanium wire materials," Materials Science and Engineering: A, vol. 527, no. 15, pp. 3301-3309, 2010.

[9] Q. Tan and G. He, "3D entangled wire reinforced metallic composites," Materials Science and Engineering: A, vol. 546, pp. 233-238, 2012.

[10] Q. Tan, P. Liu, C. Du, L. Wu, and G. He, "Mechanical behaviors of quasi-ordered entangled aluminum alloy wire material," Materials Science and Engineering: A, vol. 527, no. 1-2, pp. 38-44, 2009.

[11] J. F. Hou, H. B. Bai, and D. W. Li, "Damping capacity measurement of elastic porous wire-mesh material in wide temperature range," Journal of Materials Processing Technology, vol. 206, no. 1-3, pp. 412-418, 2008.

[12] D. W. Childs, "The space shuttle main engine high-pressure fuel turbopump rotordynamic instability problem," Journal of Engineering for Gas Turbines and Power, vol. 100, no. 1, pp. 48-57, 1977.

[13] B. Ertas, H. Luo, and D. Hallman, "Dynamic characteristics of shape memory alloy metal mesh dampers," in Proceedings of the 50th AIAA/ASME/ASCE/AHS/ASC Structures, Structural Dynamics, and Materials Conference, Palm Springs, CA, USA, June 2012.

[14] B. H. Ertas and H. Luo, "Nonlinear dynamic characterization of oil-free wire mesh dampers," Journal of Engineering for Gas Turbines and Power, vol. 130, no. 3, 2008.

[15] J. Hu, Q. Du, J. Gao, J. Kang, and B. Guo, "Compressive mechanical behavior of multiple wire metal rubber," Materials \& Design, vol. 140, pp. 231-240, 2018.

[16] B. Gadot, O. Riu Martinez, S. Rolland Du Roscoat, D. Bouvard, D. Rodney, and L. Orgéas, "Entangled single-wire NiTi material: a porous metal with tunable superelastic and shape memory properties," Acta Materialia, vol. 96, pp. 311-323, 2015.

[17] Z. Y. Ren, Q. Chen, H. Bai, and Y. Wu, "Study on damping energy dissipation characteristics of cylindrical metal rubber in nonforming direction," Advances in Materials Science and Engineering, vol. 2018, Article ID 5014789, 10 pages, 2018.

[18] P. Yang, H. Bai, X. Xue, K. Xiao, and X. Zhao, "Vibration reliability characterization and damping capability of annular periodic metal rubber in the non-molding direction," Mechanical Systems and Signal Processing, vol. 132, pp. 622-639, 2019.

[19] D. Zhang, F. Scarpa, Y. Ma, J. Hong, and Y. Mahadik, "Dynamic mechanical behavior of nickel-based superalloy metal rubber," Materials \& Design, vol. 56, pp. 69-77, 2014.

[20] H.-Y. Jiang, D.-G. Hao, Y.-H. Xia, and A.M. Ulanov, “A damping characteristics calculation method of metal dry friction isolators," Journal of Bjing Institute of Technology, vol. 17, no. 2, pp. 173-177, 2008.

[21] L. Yuyan, H. Xieqing, and M. Wenxiong, "A theoretical model and experimental investigation of a nonlinear constitutive equation for elastic porous metal rubbers," Mechanics of Composite Materials, vol. 41, no. 4, pp. 303-312, 2005. 
[22] Y. Li, X. Li, and X. Huang, "Research on factors influencing the mechanical properties of metallic rubber," Mechanics of Composite Materials, vol. 47, no. 5, pp. 571-580, 2011.

[23] C. Fengli, B. Hongbai, L. Dongwei, R. Guoquan, and L. Guozhang, "A constitutive model of metal rubber for hysteresis characteristics based on a meso-mechanical method," Rare Metal Materials and Engineering, vol. 45, no. 1, pp. 1-6, 2016.

[24] M.-J. Huang, X.-P. Dong, and G.-Q. Liu, "3D Modeling of the preparation process of metal rubber material," International Journal of Minerals, Metallurgy, and Materials, vol. 17, no. 1, pp. 75-79, 2010.

[25] B. Zhang, Z. Q. Lang, S. A. Billings, G. R. Tomlinson, and J. A. Rongong, "System identification methods for metal rubber devices," Mechanical Systems and Signal Processing, vol. 39, no. 1-2, pp. 207-226, 2013.

[26] Y. Ma, Q. Zhang, D. Zhang, F. Scarpa, B. Liu, and J. Hong, "The mechanics of shape memory alloy metal rubber," Acta Materialia, vol. 96, pp. 89-100, 2015.

[27] Y. Ma, Q. Zhang, D. Zhang, F. Scarpa, B. Liu, and J. Hong, "A novel smart rotor support with shape memory alloy metal rubber for high temperatures and variable amplitude vibrations," Smart Materials and Structures, vol. 23, no. 12, Article ID 125016, 2014.

[28] H.-K. Jeong, J.-H. Han, S.-H. Youn, and J. Lee, "Frequency tunable vibration and shock isolator using shape memory alloy wire actuator," Journal of Intelligent Material Systems and Structures, vol. 25, no. 7, pp. 908-919, 2013.

[29] P. Liu, G. He, and L. H. Wu, "Impact behavior of entangled steel wire material," Materials Characterization, vol. 60, no. 8, pp. 900-906, 2009.

[30] Y. Wu, S. Li, H. Bai, L. Jiang, and H. Cheng, "Experimental and constitutive model on dynamic compressive mechanical properties of entangled metallic wire material under lowvelocity impact," Materials, vol. 13, no. 6, p. 1396, 2020.

[31] P. Wang, G. Y. Zhang, Y. J. Gao, J. W. Ding, and X. L. Lang, "Application of metal-rubber dampers in vibration reduction system of an airborne electro-optical pod," Zhendong $Y u$ Chongji/Journal of Vibration and Shock, vol. 33, no. 5, pp. 193-199, 2014.

[32] H. Gao, C. Xiong, J. Yin, and H. Deng, "Research on dynamic accumulation effect and constitutive model of aluminum foams under dynamic impact," International Journal of Metalcasting, vol. 13, no. 1, pp. 146-157, 2019.

[33] H. Yu, Z. Guo, B. Li, G. Yao, H. Luo, and Y. Liu, "Research into the effect of cell diameter of aluminum foam on its compressive and energy absorption properties," Materials Science and Engineering: A, vol. 454-455, pp. 542-546, 2007.

[34] Q. M. Li, I. Magkiriadis, and J. J. Harrigan, "Compressive strain at the onset of densification of cellular solids," Journal of Cellular Plastics, vol. 42, no. 5, pp. 371-392, 2006.

[35] J. Miltz and G. Gruenbaum, "Evaluation of cushioning properties of plastic foams from compressive measurements," Polymer Engineering and Science, vol. 21, no. 15, pp. 10101014, 1981. 